$$
\text { كلية الطب الأدوية و السموم البيطرية البية - جامعث - سوريا }
$$

\title{
EFFECT OF WATERY EXTRACT OF ARTEMISIA HERBA ALBA ON BLOOD GLUCOSE LEVEL AND BODY WEIGHT IN ALLOXAN - DIABETIC RABBITS
}

(With 4 Tables and 2 Figures)

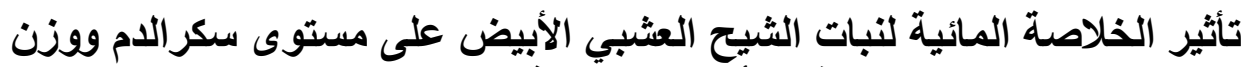

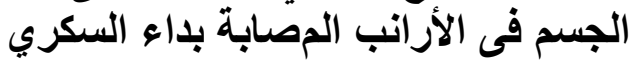

(Received at 1/6/2010)

\section{بيان عفوف ، عبد الرزاق حموية ، أسعد العبل}

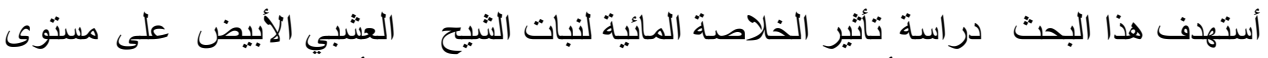

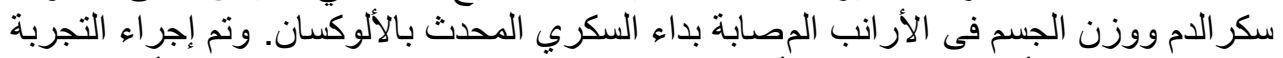

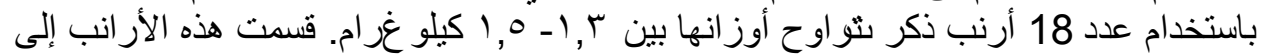

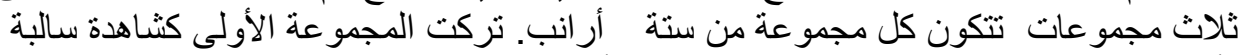

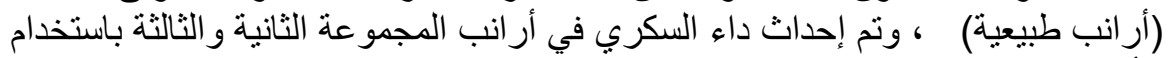

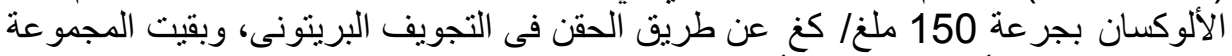

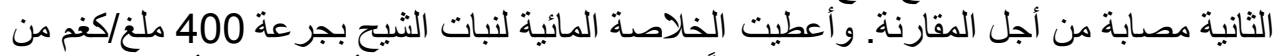

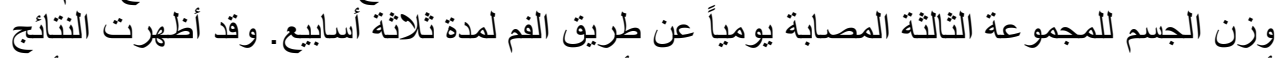

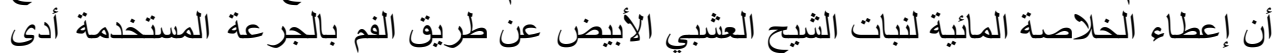

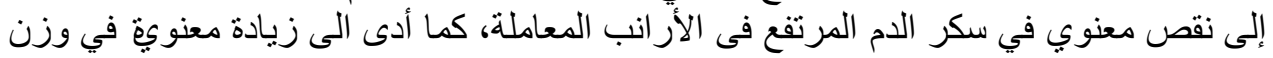

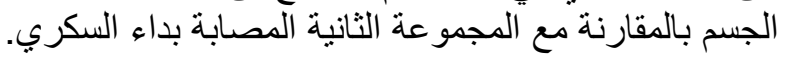

\section{SUMMARY}

The objective of this study was to investigate the effect watery extract of Artemisia herba alba on blood glucose level and body weight in alloxan diabetic rabbits. The experiment was carried out on 18 male rabbits $(1.3-$ $1.5 \mathrm{~kg} / \mathrm{b}$.wt.) distributed into 3 groups, of 6 animals each. The $1^{\text {st }}$ group was kept as normal control, while the $2^{\text {nd }}$ and $3^{\text {rd }}$ groups were rendered diabetic by intraperitoneal injection of alloxan $\left(150 \mathrm{mg} / \mathrm{kg}\right.$ b.wt.). The $2^{\text {nd }}$ group was left as diabetic control, while the $3^{\text {rd }}$ group was given orally the watery extract of Artemisia herba in a dose of $400 \mathrm{mg} / \mathrm{kg}$ b.wt./day for 3 weeks. Blood samples were collected for determination of glucose levels and rabbits were weighed at the beginning and end of the experiment. The obtained results showed that oral administration of Artemisia herba watery extract to diabetic rabbits significantly $(\mathrm{p}<0.05)$ decreased the high blood glucose level and increased body weight of the treated diabetic rabbits, as 
compared to the diabetic control group. In conclusion Artemisia herba watery extract produces antidiabetic effect in alloxan - diabetic rabbits and it may be beneficial for patients who suffer from diabetes mellitus.

Key words: داء السكرى ، الالوكسان ، نبات الثبح العشبى الأبيض ، الأرانب

\section{INTRODUCTION}

مقدمـة

يعرف داء السكري Diabetes mellitus على أنه حالة وراثية أو مكتسبة يطلق عليها

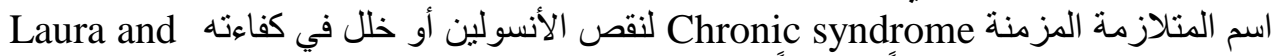

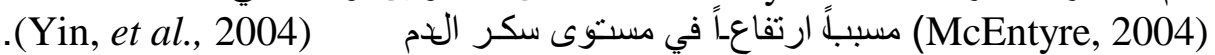

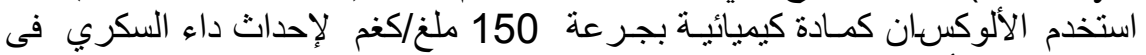

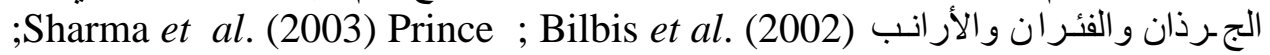
et al. (2004)

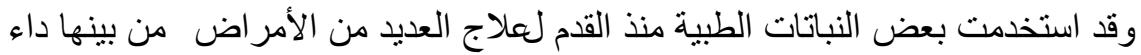

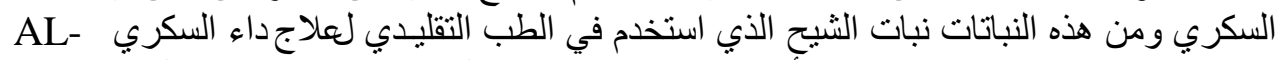

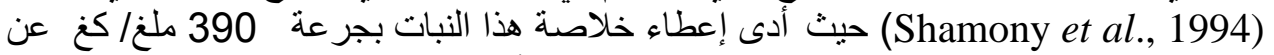
طريق الوريد للجرذان المصابة بداء السكري المحدث بالألوكسان إلى انخفاض مستوى سكر الداء

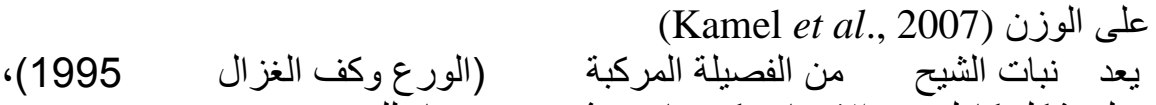

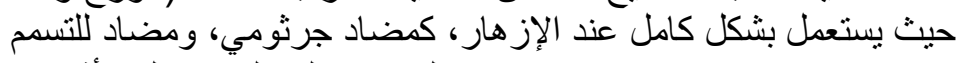

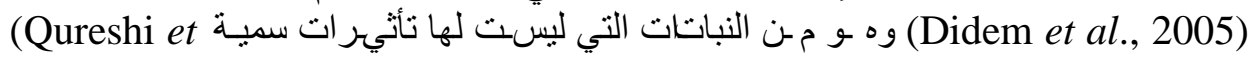

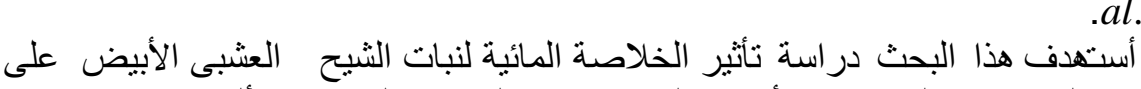
$. a l ., 1990)$

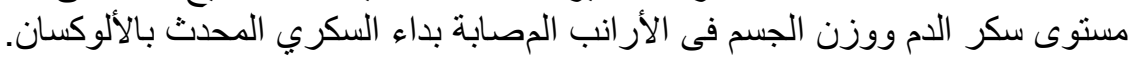

\section{MATERIALS and METHODS المواد وطر ائق العمل}

أولاً: الخلاصة المائية للنبات:

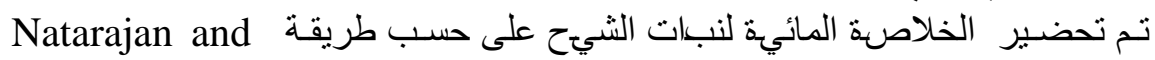

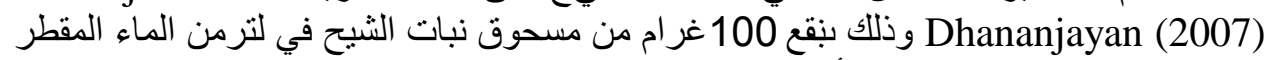

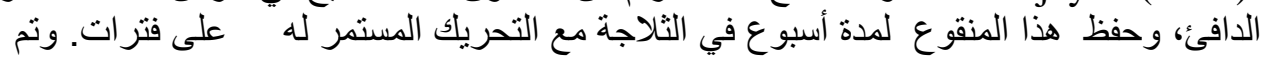

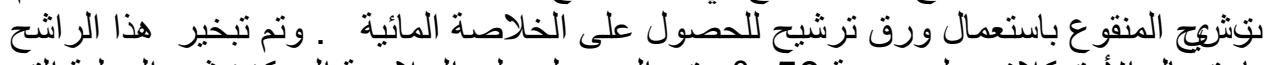

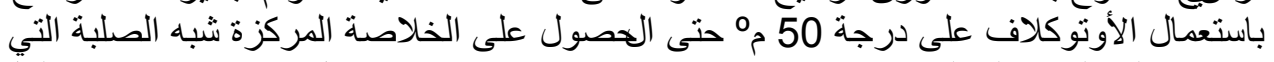

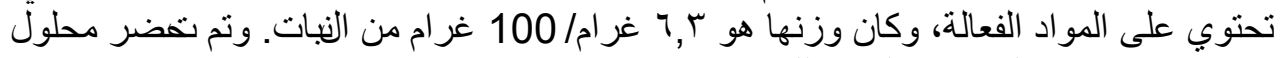
تركيزه •2\% من الخلاصة المائية للنبات.

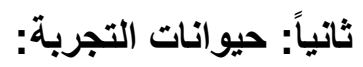




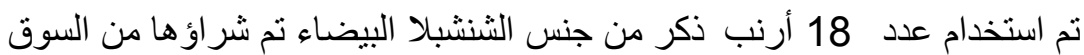

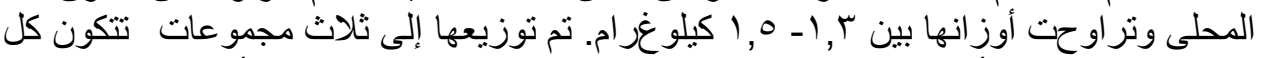

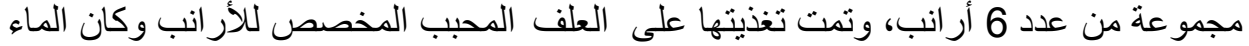
متاح لهم بكمية كافية طيلة فترة التجربة.

ثالثاً: إحداث داء السكري بالألوكسان:

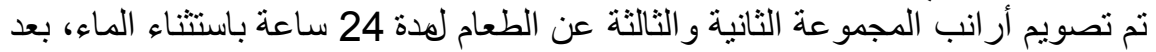

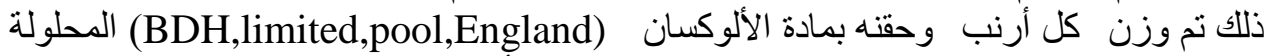

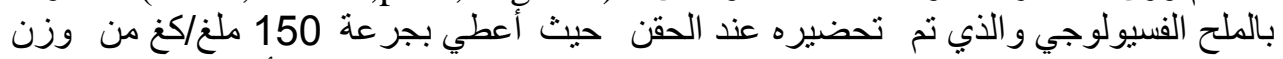

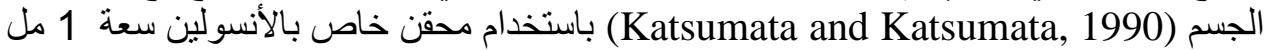

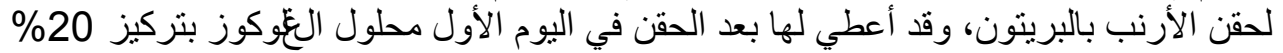

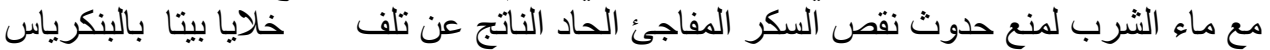
وتم وضع العلف بعد الحقن. وقد تم التأكد من إحداث داء السكري (Ananthan et al., 2003)

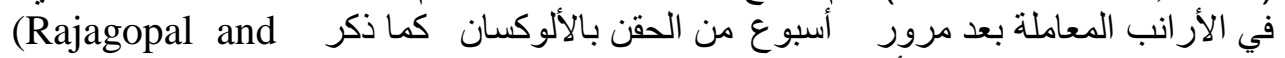

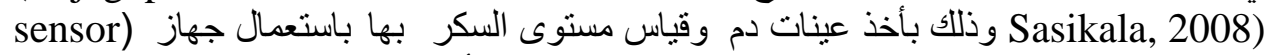

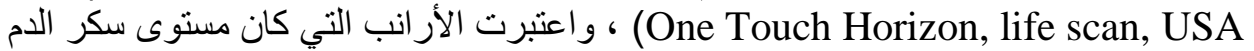

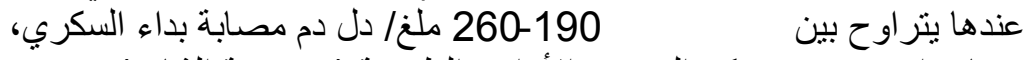

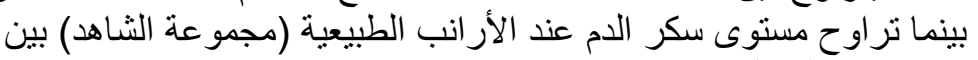

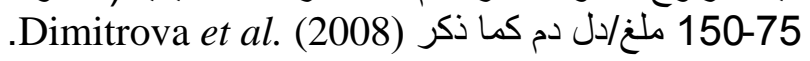

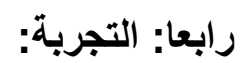
تم تقسيم مجموعات التجربة: أر انب التجربة على النحو التالي:

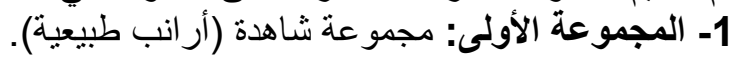
2- المجموعة الثانية: محقونة بالألوكسان (مصابة بداء السكري) نركت بدونة بلدون معاملة حتى نهاية

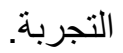

3- المجموعة الثالثة: محقونة بالألوكسان (مصابة بداء السكري) و معاملة بالخلاصة المائية

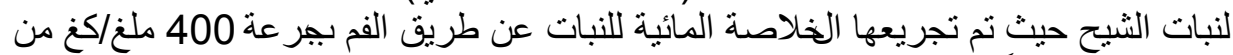

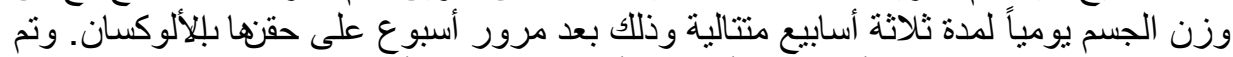

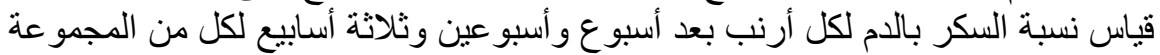

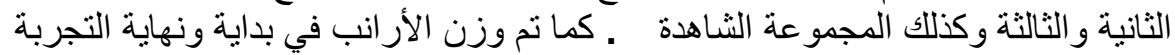
وحساب فرق الوزن ومتوسط معدل الزيادة أو النقصان في أوزان الآر انب.

استخدم اختبار التباين الأحادي One-Way ANOVA test لتحليل التحليل النتائج التي حصلنا خامساً: التحليل الإحصائي:

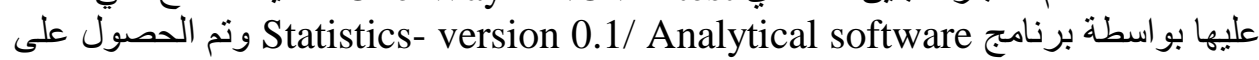
المنوسط الحسابي و الخطأ المعياري للمتوسط واحتساب الفرق بين المجمو عنين معنوي عند قيمة

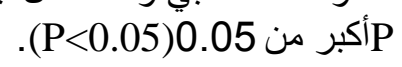

\section{RESULTS}

النتائج 


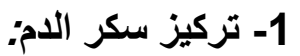

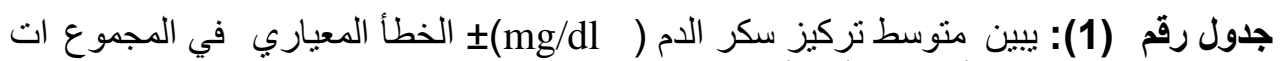
الثناث للأر انب وتثأثير أعطاء بالخلاصة المائية لنبات الثيح عن طريق الفم على الثي

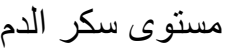

\begin{tabular}{|c|c|c|c|}
\hline $\begin{array}{c}\text { المجموعة الثالثة } \\
\text { Diabetic given } \\
\text { Artemisia } \\
\text { watery extract }\end{array}$ & $\begin{array}{c}\text { المجموعة الثانية } \\
\text { Experimentally } \\
\text { Diabetic }\end{array}$ & $\begin{array}{c}\text { المجموعة الأولى } \\
\text { Control } \\
\text { normal }\end{array}$ & د الدمبط تركيز سكر \\
\hline 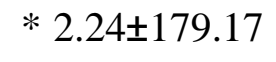 & $3.35 \pm 195.50$ & $2.37 \pm 135.33$ & بعد أسبوع \\
\hline$* * 4.11 \pm 164.00$ & $3.43 \pm 200.83$ & $1.7 \pm 141.33$ & بعد أسبو عين \\
\hline$* * 3.64 \pm 157.83$ & $5.27 \pm 204.50$ & $1.8 \pm 140.67$ & بعد ثلاثة أسابيع \\
\hline
\end{tabular}

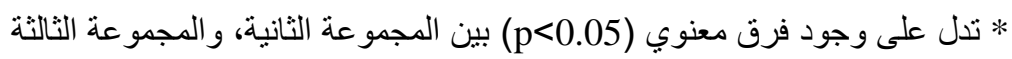
المعاملة بالخلاصة المائية لنبات الثيح. * * تدل على وجود فرق معنوي جداً (

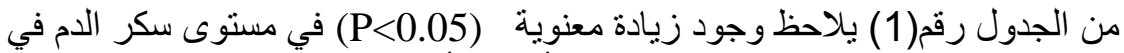

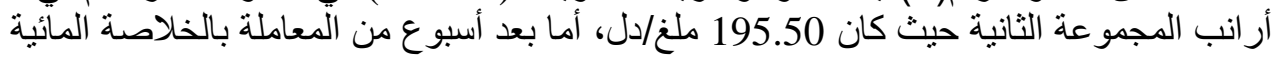

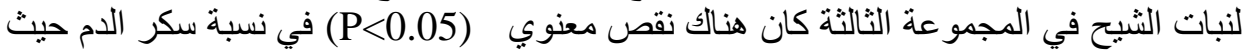

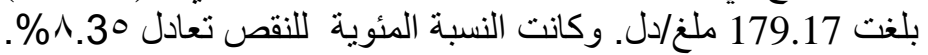

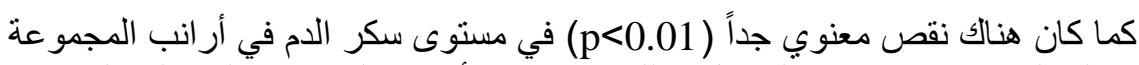

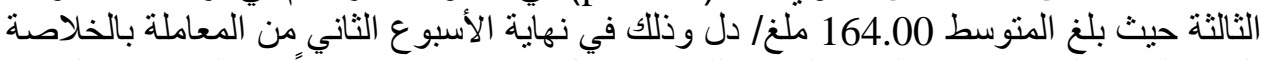

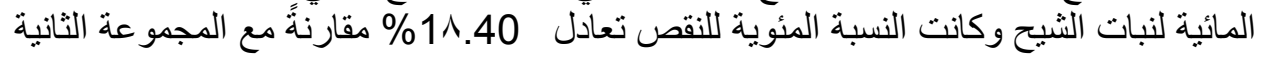

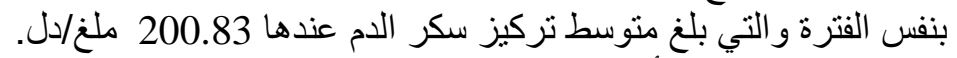

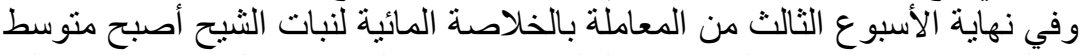

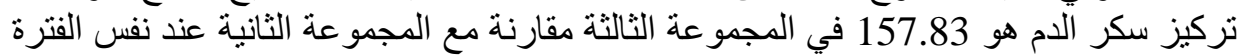

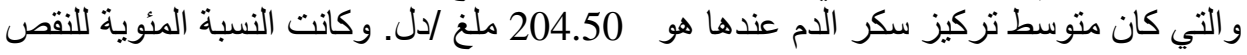

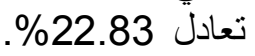

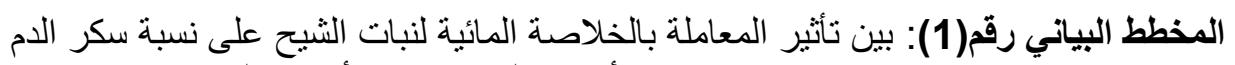
mg/dl في مجمو عات أر انب التجربة في الأسابيع الثلاثة 


\section{Assiut Vet. Med. J. Vol. 56 No. 126 July 2010}

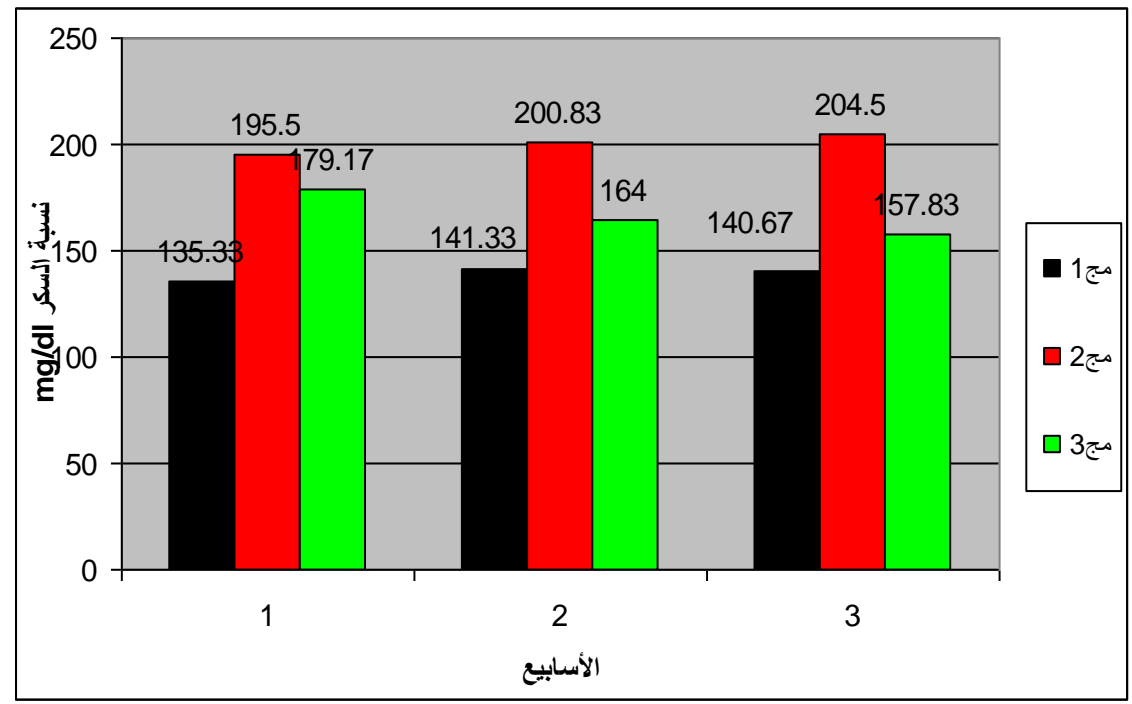

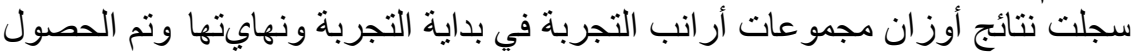

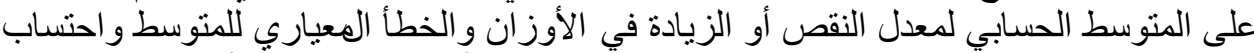

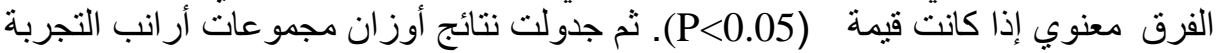

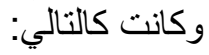

الجدول رقم(2): يوضح أوزان أرانب مجمو عات التجربة في بداية التجربة

\begin{tabular}{|c|c|c|c|}
\hline \multicolumn{3}{|c|}{ وزن الأرنب في المجموعة الواحدة/غ/ في بداية التجربة } & \multirow{2}{*}{ رقم الأرنب } \\
\hline مج3 & مج2 & مج1 الثاهدة & \\
\hline 1440 & 1400 & 1470 & 1 \\
\hline 1324 & 1430 & 1500 & 2 \\
\hline 1420 & 1350 & 1400 & 3 \\
\hline 1331 & 1375 & 1395 & 4 \\
\hline 1482 & 1460 & 1330 & 5 \\
\hline 1380 & 1365 & 1410 & 6 \\
\hline $25.54 \pm 1396$ & $17.11 \pm 1396$ & $24.55 \pm 1417$ & المتوسط \\
\hline
\end{tabular}

جدول رقم(3): يوضح أوزان أرانب مجمو عات التجربة في نهاية التجربة

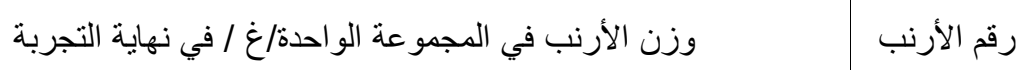


Assiut Vet. Med. J. Vol. 56 No. 126 July 2010

\begin{tabular}{|c|c|c|c|}
\hline السكري + مجلاصدة مصابة بداء النبات & مجاء مصابة السكري & أرانب طبيعية الثاهد & \\
\hline 1620 & 1308 & 1710 & 1 \\
\hline 1461 & 1343 & 1678 & 2 \\
\hline 1593 & 1281 & 1610 & 3 \\
\hline 1472 & 1315 & 1562 & 4 \\
\hline 1634 & 1372 & 1503 & 5 \\
\hline 1510 & 1303 & 1579 & 6 \\
\hline $31.30 \pm 1548$ & $13.18+1320$ & $31.42+1607$ & المتوسط المعياري \\
\hline
\end{tabular}

المخطط البياني رقم (2): يبين معدل الفرق في أوزان الأر انب عند بداية ونهاية التجربة وبعد

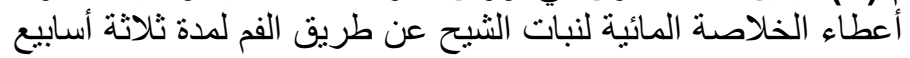

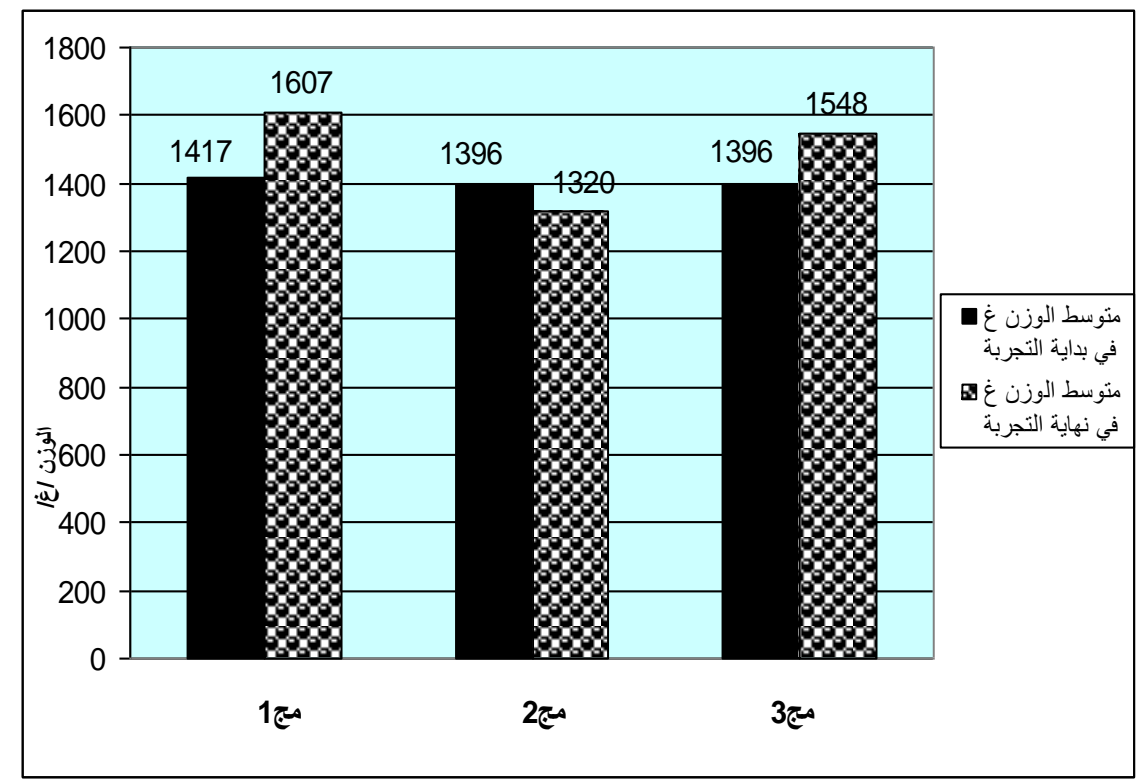

جدول رقم ( 4): يبين معدل الفرق في أوزان الأر انب بين بداية ونهاية التجربة وبعد أعطاء

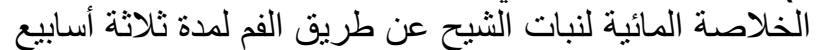

\begin{tabular}{|c|c|c|}
\hline معدل الزيادة في وز & وزن الأرانب/غ / معدان في & معدل الزيادة في وزن إغان/ \\
\hline
\end{tabular}




\section{Assiut Vet. Med. J. Vol. 56 No. 126 July 2010}

\begin{tabular}{|c|c|c|c|}
\hline السكري + خلابة بداء النبات & بداء السكري مصابة & أرانب طبيعية الثاهد & \\
\hline 180 & 92 & 240 & 1 \\
\hline 137 & 87 & 178 & 2 \\
\hline 173 & 69 & 210 & 3 \\
\hline 141 & 60 & 167 & 4 \\
\hline 152 & 88 & 173 & 5 \\
\hline 130 & 62 & 169 & 6 \\
\hline$* * 8.27 \pm 152.13$ & $5.83 \pm 76.33$ & $11.97 \pm 189.50$ & 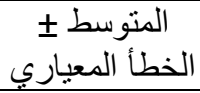 \\
\hline
\end{tabular}

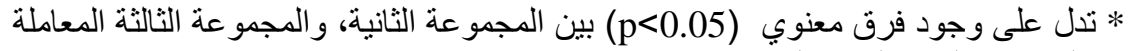

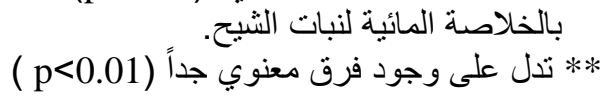

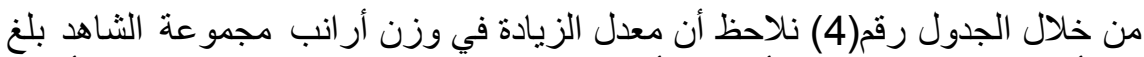

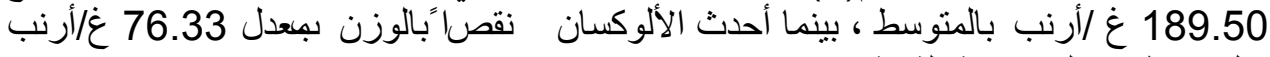
بالمتوسط في المجموعة الثانية

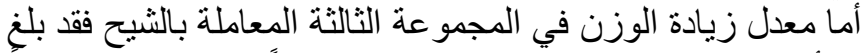

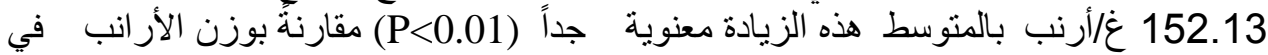

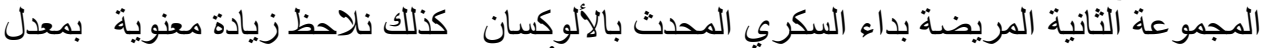

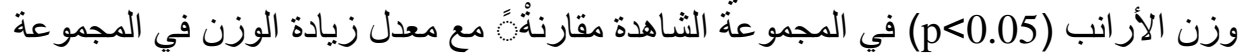
الثالثة المعاملة بالثيح. و المخطط البياني رقم(3) ييين ذلك.

المخطط البياني رقم(3): ييين معدل الزيادة أو النقصان في أوزان الأرانب في نهاية التجربة بعد التاني

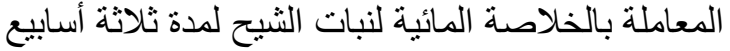

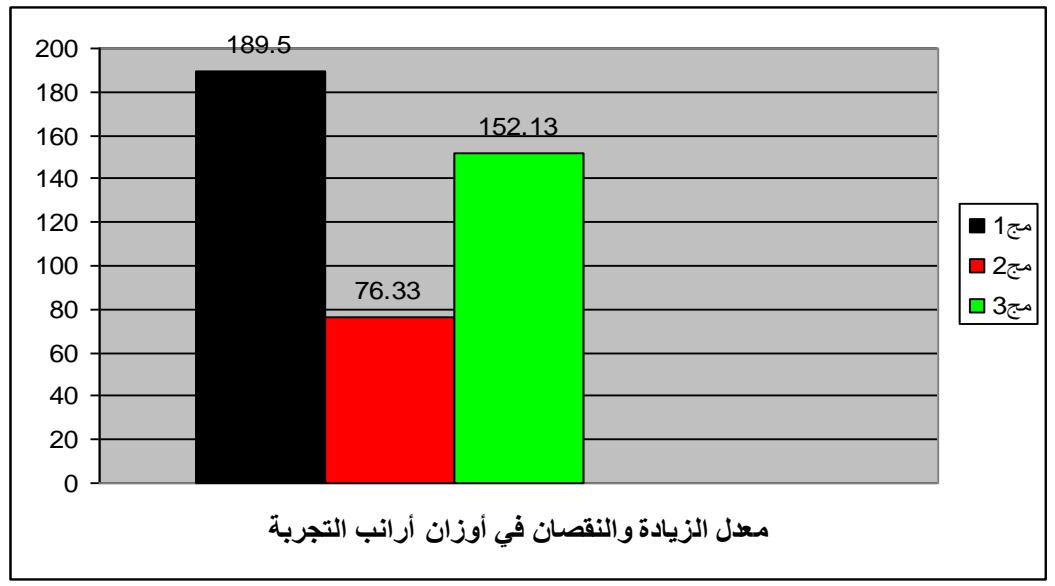

\section{DISCUSSION}

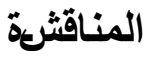




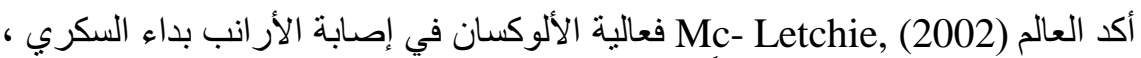

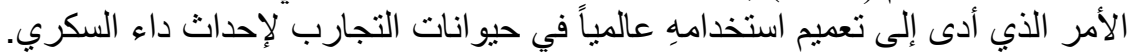

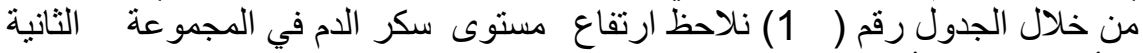

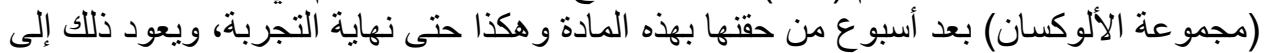

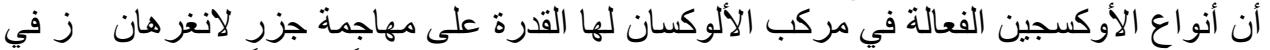

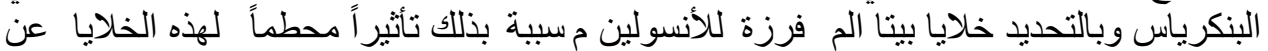
طريق التلف التأكسدي (Tiedge et al., 1997) يعمل الألوكسان على زيادة مستوى الأنسولين بالدم في الساعات الأولى بعد الحقن

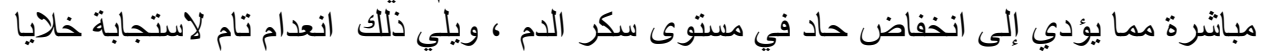

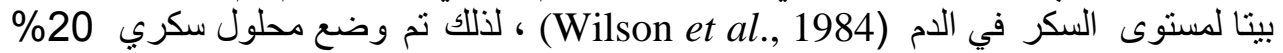

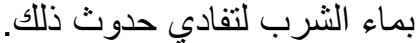

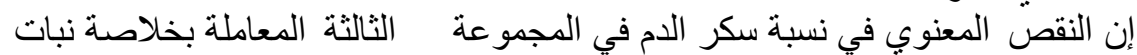

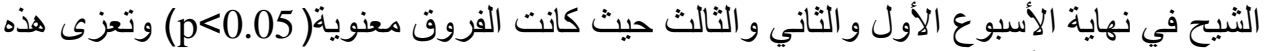

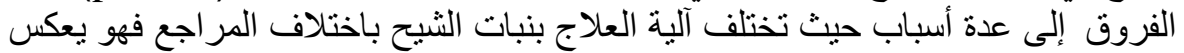

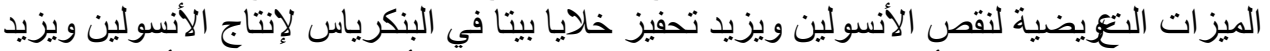

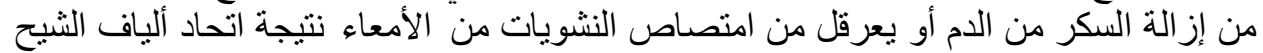
معها أو يمنع إعادة امنصاص السكر الس من الر اثتح الكلوي (Didem et al., 2005).

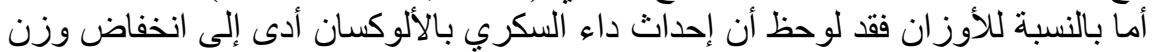

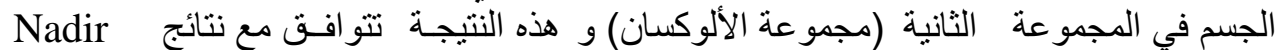

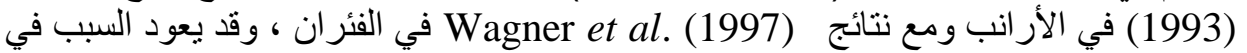

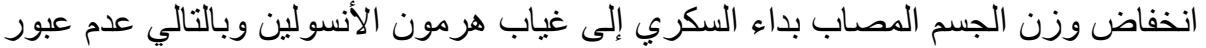

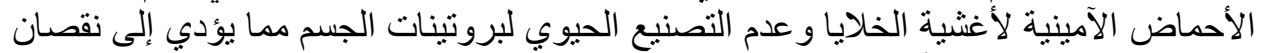

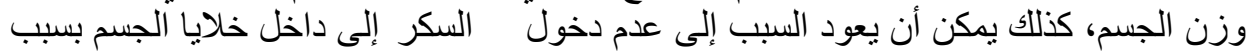

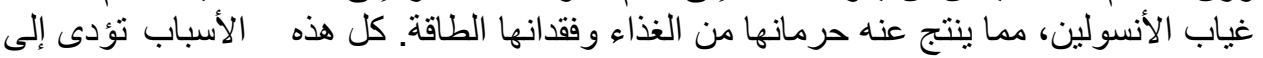
حدوث نقص في وزن الجسم (Holm, 1997).

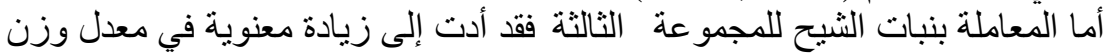

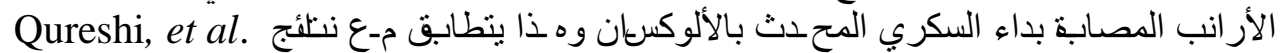

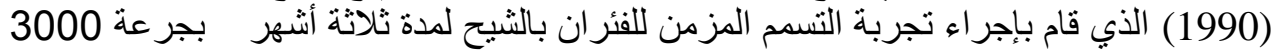

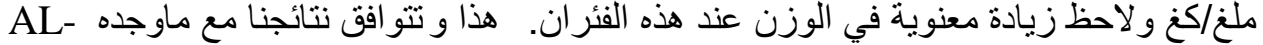

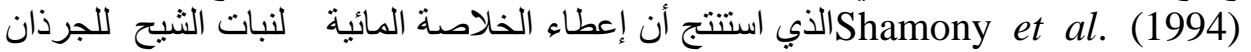

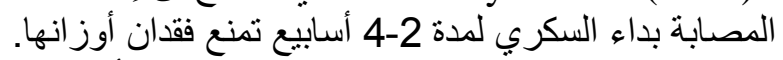

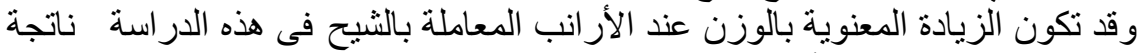

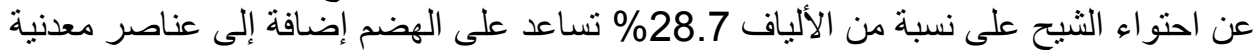

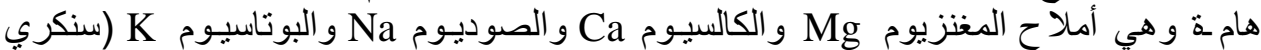
1987,

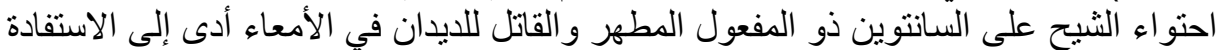

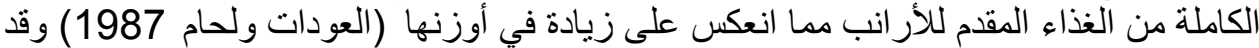

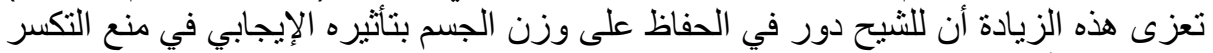

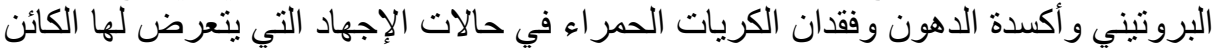

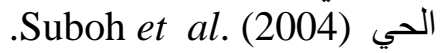



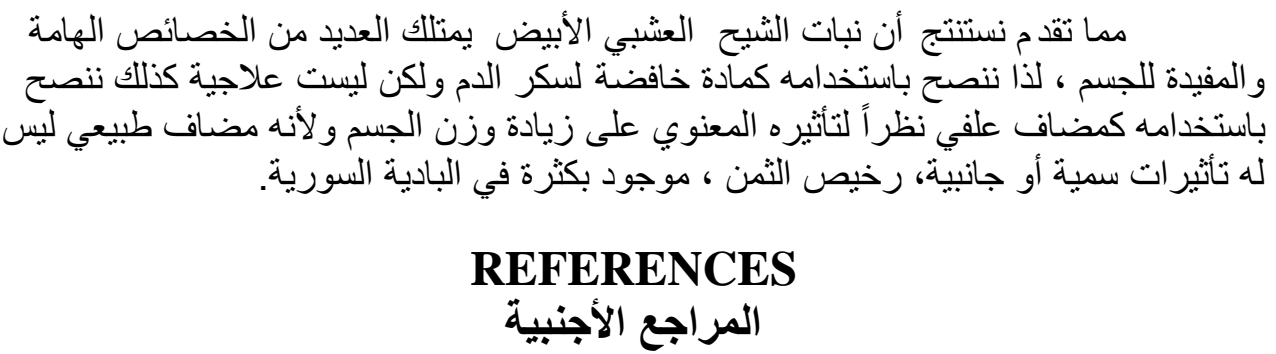

AL-Shamony, L.A.; AL-Khazraji, S.M. and Twaij, H.A. (1994): Hypoglycaemic effect of Artemisia herba alba. II. Effect of alcoholic extract on some blood parameters in diabetic animals. J. Ethnopharmacol. 22; 43(3):167-171.

Ananthan, R.; Latha, M.; Ramakumar, K.M.; Pari, L. and Narmatha, B. (2003): Effect of Gymnema montanum Leaves on serum and tissue lipids in alloxan diabetic rats. 4: 183-189.

Bilbis, L.S.; Shehu, R.A. and Abubakar, M.G. (2002): Hypoglycemic and hypolipidemic effects of aqueous extract of Arachis hypogaea in normal and alloxan induced diabetic rats. Phytomed., 9(6): 553-555.

Didem, T.; Al Asefer, M.; Gulsah, A.; Keles, M. and Ayhan, T. (2005): Hypoglycemic effect of watery extract of Artemisia herba alba in experimental hyperglycemic rats. Erzurum., Turkey, 20: 252 -256.

Dimitrova, S.S.; Georgiev, I.P.; Kanelov, I.N.; Iliev, Y.I. and Taner, S.I. (2008): Intravenous glucose tolerance test and glucose pharmacokinetic parameters in rabbits. Bulg. J. Vet. Med., 11(3): 161-169.

Hermansen, K.; Ndergaard, M.; Lars, H.; Carstensen, M. and Brock, B. (2001): Beneficial effects of a soy based dietary supplement on lipid levels. Diab. Care, 24: 228-233.

Holm, B. (1997): Diabetes mellitus in the dog. Eur. J. Comp. Anim. Pract., 7: 61-66.

Kamel, M.; Mosalamey, A. and Hamzah, N. (2007): The hypolipidemic effect of Siberian Artemisia (A. herba alba) in alloxan induced diabetic rats. J. Basic App. Sci. 4(2): 57-62.

Katsumata, K. and Katsumata, Y. (1990): The potentiating effect of the simultaneous administration of tolbutamide, glibenclamide, and gliclazide on the development of alloxan - induced diabetes in rats. Hom. Metab. Res., 22: 51-52.

Laura, D. and McEntyre, J.R. (2004): The Genetic Landscape of Diabetes. National Library of Medicine. U.S.A. 
Mc-Letchie, N.G. (2002): Alloxan diabetes: a discovery to induce a minor one. J.R. Coll. Physi., 32 (2): 134-142.

Nadir, H.H. (1993): Tissue glutathione alterations in chronic alloxan induced diabetes in rabbits: Insulin therapy. Iraqi J. Vet. Sci., 6: 21-23.

Natarajan, B. and Dhananjayan, (2007): Pharmacological effects of Trigonella faenum graecum seeds on various isolated perfused smooth muscle preparations. Pharmacol. Magaz.., 3 (10): 77-80.

Prince, D.S.; Kamalakkannan, N. and Menon, V.P. (2004): Antidiabetic and antihyperlipidemic effect of alcoholic extract of Syzigium cumin seeds in alloxan induced diabetic albino rats. J. Ethnopharmacol., 91 (203): 209-213.

Qureshi, S.; Ageel, A.M.; Al-Yahya, M.A.; Tariq, M.; Mossa, J.S. and Salah, A.H. (1990): Preliminary toxicity studies on ethanol extract of the aerial parts of Artemisia Abyssinia and A. Inculta in mice. Phytomed., 4: 157-162.

Rajagopal, K. and Sasikala, K. (2008): Antidiabetic activity of hydroethanolic extracts of Nymphaea Stellata flowers in normal and alloxan - induced diabetic rats. Afr. J. Pharmac., 6: 173-178.

Sharma, S.B.; Nasir, A.; Prabhu, K.M.; Murthy, P.S. and Dev, G. (2003): Hypoglycemic and hypolipidemic effect of ethanolic extract of seeds of Eugenia jambolana in alloxan-induced diabetic rabbits. J. Ethnopharmacol., 85 (2-3): 201-206.

Suboh, S.M.; Biloto, Y.Y. and Aburjai, T.A. (2004): Protective effect of some selected medicinal plants against protein degration, lipid peroxidation and deformability loss of oxidative stressed human erythrocytes. J. Ethnopharmacol. 86 (4): 280-284.

Tiedge, M.; Lortz, S.; Drinkgern, J. and Lenzen, S. (1997): Relation between antioxidant enzyme gene expression and antioxidative defense status of insulin producing cells. Diabetes, 46: 1733-1742.

Wilson, G.L.; Patton, N.J.; McCord, J.M.; Mullins, D.W. and Mossman, B.T. (1984): Mechanisms of streptozotocin and alloxan- induced damage in rat $\beta$ cells. Dialectologies., 27(6): 587-591.

Wagner, J.D.; Cefalu, W.T.; Anthony, M.S.; Litwak, K.N.; Zhang, L. and Clarleson, T.B. (1997): Dietary soy protein and estrogen replacement therapy improve cardiovascular risk factors and decrease aortic cholesterol. Metabolism, 46(6): 698-705.

Yin, X.Z.; Quan, J.S.; Takemichi, K. and Mukoto, T. (2004): Antiatherosclerotic effect of soybean isoflavones and soy saponins in diabetic rats. Za-Zhi., 35(1): 26-28. 


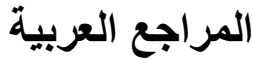

العودات ، محد ولحام ، جورج (1987): النباتات الطبية واستعمالاتها كلية الصيدلة ، منشور ات جامعة دمثق. الورع ، حسان بشير وكف الغزال، رامي ( 1995): النباتات الطبية والعطرية، كلبة الزراعة، منشور ات جامعة البعث.

سنكري، محد ننبير (1987): بيئات ونباتات مر اعي المناطق الجافة وشديدة الجفاف السورية

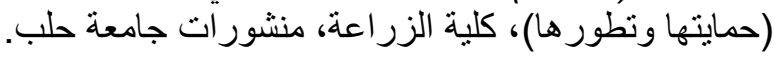

